\title{
Capillary Break-up Rheometry of Low-Viscosity Elastic Fluids
}

\author{
Lucy E. Rodd ${ }^{1,3}$, Timothy P. Scott ${ }^{3}$ \\ Justin J. Cooper-White ${ }^{2}$, Gareth H. McKinley ${ }^{3}$ \\ ${ }^{1}$ Dept. of Chemical and Biomolecular Engineering, \\ The University of Melbourne, VIC 3010, Australia \\ ${ }^{2}$ Division of Chemical Engineering, \\ The University of Queensland, Brisbane, QLD 4072, Australia \\ ${ }^{3}$ Hatsopoulos Microfluids Laboratory, Dept. of Mechanical Engineering, \\ Massachusetts Institute of Technology, Cambridge, MA 02139, USA
}

July 28, 2004

\begin{abstract}
We investigate the dynamics of the capillary thinning and break-up process for low viscosity elastic fluids such as dilute polymer solutions. Standard measurements of the evolution of the midpoint diameter of the necking fluid filament are augmented by high speed digital video images of the break up dynamics. We show that the successful operation of a capillary thinning device is governed by three important time scales (which characterize the relative importance of inertial, viscous and elastic processes), and also by two important length scales (which specify the initial sample size and the total stretch imposed on the sample). By optimizing the ranges of these geometric parameters, we are able to measure characteristic time scales for tensile stress growth as small as 1 millisecond for a number of model dilute and semi-dilute solutions of polyethylene oxide (PEO) in water and glycerin. If the aspect ratio of the sample is too small, or the total axial stretch is too great, measurements are limited, respectively, by inertial oscillations of the liquid bridge or by the development of the well-known beads-on-a-string morphology which disrupt the formation of a uniform necking filament. By considering the magnitudes of the natural time scales associated with viscous flow, elastic stress growth and inertial oscillations it is possible to construct an "operability diagram" characterizing successful operation of a capillary break-up extensional rheometer. For Newtonian fluids, viscosities greater than approximately $70 \mathrm{mPa} . \mathrm{s}$ are required; however for dilute solutions of high molecular weight polymer the minimum viscosity is substantially lower due to the additional elastic stresses arising from molecular extension. For PEO of molecular weight $10^{6} \mathrm{~g} / \mathrm{mol}$, it is possible to measure relaxation times of order $1 \mathrm{~ms}$ in dilute polymer solutions of viscosity $2-10 \mathrm{mPa}$.s.
\end{abstract}




\section{Introduction}

Over the past 15 years capillary break-up elongational rheometry has become an important technique for measuring the transient extensional viscosity of non-Newtonian fluids such as polymer solutions, gels, food dispersions, paints, inks and other complex fluid formulations. In this technique, a liquid bridge of the test fluid is formed between two cylindrical test fixtures as indicated schematically in figure 1(a). An axial step-strain is then applied which results in the formation of an elongated liquid thread. The profile of the thread subsequently evolves under the action of capillary pressure (which serves as the effective 'force transducer') and the necking of the liquid filament is resisted by the combined action of viscous and elastic stresses in the thread.

In the analogous step-strain experiment performed in a conventional torsional rheometer, the fluid response following the imposition of a step shearing strain (of arbitrary magnitude $\gamma_{0}$ ) is entirely encoded within a material function referred to as the relaxation modulus $G\left(t, \gamma_{0}\right)$. By analogy, the response of a complex fluid following an axial step strain is encoded in an apparent transient elongational viscosity function $\eta_{E}(\dot{\varepsilon}, t)$ which is a function of the instantaneous strain

rate, $\dot{\varepsilon}$ and the total Hencky strain $\left(\varepsilon=\int \dot{\varepsilon} d t^{\prime}\right)$ accumulated in the material. An important factor complicating the capillary break-up technique is that the fluid dynamics of the necking process evolve with time and it is essential to understand this process in order to extract quantitative values of the true material properties of the test fluid. Although this complicates the analysis and results in a time-varying extension rate, this also makes the capillary thinning and breakup technique an important and useful tool for measuring the properties of fluids that are used in free-surface processes such as spraying, roll-coating or ink-jetting. Well-characterized model systems (based on aqueous solutions of polyethylene oxide ) have been developed for studying such processes in the past decade (Dontula et al. 1998; Harrison \& Boger, 2000) and we study the same class of fluids in the present study.

Significant progress in the field of capillary break-up rheometry has been made in recent years since the pioneering work of Entov and co-workers (Basilevskii et al. 1990; 1997). Capillary thinning and break-up has been used to measure quantitatively the viscosity of viscous and elastic fluids (McKinley \& Tripathi, 1999; Anna \& McKinley, 2001); explore the effects of salt on the extensional viscosity for important drag-reducing polymers and other ionic aqueous polymers (Stelter et al; 2000, 2002), monitor the degradation of polymer molecules in elongational flow (Basilevskii et al. 1997) and the concentration dependence of the relaxation 
time of polymer solutions (Basilevskii et al. 2001). The effects of heat or mass transfer on the time-dependent increase of the extensional viscosity resulting from evaporation of a volatile solvent in a liquid adhesive have also been considered (Tripathi et al. 1999); and more recently the extensional rheology of numerous inks and paint dispersions have been studied using capillary thinning rheometry (Willenbacher, 2004). The relative merits of the capillary break-up elongational rheometry technique (or CABER) and filament stretching elongational rheometry (or FISER) have been discussed by McKinley (2000) and a detailed review of the dynamics of capillary thinning of viscoelastic fluids is provided elsewhere (McKinley, 2005).

Measuring the extensional properties of low-viscosity fluids (with zero-shear-rate viscosities of $\eta_{0} \leq 100 \mathrm{mPa} . \mathrm{s}$, say) is a particular challenge. Fuller and coworkers (1987) developed the opposed jet rheometer for studying low viscosity non-Newtonian fluids, and this technique has been used extensively to measure the properties of various aqueous solutions (see for example Hermansky et al. 1995; $\mathrm{Ng}$ et al. 1996). Large deformation rates (typically greater than $1000 \mathrm{~s}^{-1}$ ) are required to induce significant viscoelastic effects, and at such rates inertial stresses in the fluid can completely mask the viscoelastic stresses resulting from molecular deformation and lead to erroneous results (Dontula et al. 1997). Analysis of jet break-up (Schümmer \& Tebel, 1983; Christanti \& Walker, 2001) and drop pinch-off (Amarouchene et al. 2001; Cooper-White et al. 2002) have also been proposed as a means of studying the transient extensional viscosity of dilute polymer solutions. After the formation of a neck in the jet or in the thin ligament connecting a falling drop to the nozzle, the dynamics of the local necking processes in these geometries is very similar to that in a capillary break-up rheometer. However, the location of the neck or 'pinch-point' is spatially-varying and high speed photography or videoimaging is required for quantitative analysis. One of the major advantages of the CABER technique is that the minimum radius is constrained by geometry and by the initial step-strain to be close to the midplane of the fluid thread, unless very large axial strains are employed and gravitational drainage becomes important (Kolte \& Szabo, 1999).

For low viscosity non-Newtonian fluids such as dilute polymer solutions, the filament thinning process in CABER is also complicated by the effects of fluid inertia which can lead to the well-known beads-on-a-string morphology (Goldin et al. 1969; Li \& Fontelos, 2003). Stelter et al. (2000) note that such processes prevent the measurement of the extensional viscosity for some of their lowest viscosity solutions. With the increasingly widespread adoption of the CABER technique it becomes important to understand what range of working fluids can be 
studied in such instruments. If the fluid is not sufficiently viscous then the liquid thread undergoes a rapid capillary break-up process before the plates are completely separated. The subsequent thinning of the thread can thus not be monitored. The threshold for onset of this process depends on the elongational viscosity of the test fluid and is frequently described qualitatively as 'spinnability' or 'stringiness'. The transient elongational stress growth in the test fluids depends on the concentration and molecular weight of the polymeric solute as well as the background viscosity and thermodynamic quality of the solvent. In the present note we investigate the lower limits of the CABER technique using dilute solutions of polyethylene oxide (PEO) in water and water-glycerol mixtures. In order to reveal the dynamics of the break-up process we combine high-speed digital video-imaging with the conventional laser micrometer measurements of the midpoint radius $R_{\text {mid }}(t)$. We explore the consequences of different experimental configurations and the roles of solvent viscosity and polymer concentration. The results can be interpreted in terms of an 'operability diagram' based on the viscous and elastic time scales governing the filament thinning process.

\section{Experimental Methods and Dimensionless Parameters}

\section{$2.1 \quad$ Fluids}

In this study we have focused on aqueous solutions of a single nonionic polymer; polyethylene oxide (PEO; Aldrich) with molecular weight $M_{w}=2.0 \times 10^{6} \mathrm{~g} / \mathrm{mol}$. Solutions were prepared by mixing the polymer into deionized water at concentrations of $0.10 \mathrm{wt} \%$, and $0.30 \mathrm{wt} \%$ using magnetic stirrers at slow/moderate speed settings. In order to explore the effects of the background solvent viscosity, an additional solution with $0.10 \mathrm{wt} \%$ PEO dissolved in a 50/50 water/glycerol water mixture was also prepared. Additional experiments exploring the role of PEO concentration in the Capillary Break-up Rheometer have been performed by Neal \& Braithwaite (2003). The results of progressive dilution of a high molecular weight polystyrene dissolved in oligomeric styrene have also been investigated recently using capillary break-up rheometry by Clasen et al. (2004). In this latter study, the solvent viscosity of the oligomer is $\eta_{s} \geq 40$ Pa.s; these solutions are therefore significantly more viscous than the aqueous solutions discussed in the present work. 
The important physiochemical and rheological properties of the test fluids are summarized in table 1. For PEO, the characteristic ratio is $C_{\infty}=4.1$ (Brandrup et al. 1997), the repeat unit mass is $m_{0}=44 \mathrm{~g} / \mathrm{mol}$ and the average bond length is $l=0.147 \mathrm{~nm}$. The mean square size of an unperturbed Gaussian coil is $\left\langle R^{2}\right\rangle_{0}=C_{\infty}\left(3 M_{w} / m_{0}\right) l^{2}$ and we thus obtain $c^{*}=M_{w} /\left(N_{A}\left\langle R^{2}\right\rangle_{0}^{3 / 2}\right) \approx 2.53 \times 10^{-3} \mathrm{~g} / \mathrm{cm}^{3}$ for this molecular weight.

However, water is known to be a good solvent for PEO, so that the polymer coils are extended beyond the random coil configuration and the above expression is an over estimate of the coil overlap concentration. Tirtaatmadja et al. (2004) summarize previous reported values of the intrinsic viscosity for numerous high molecular weight PEO/water solutions. The measurements can be well described by the following Mark-Houwink expression

$$
[\eta]=0.072 M_{w}^{0.65}
$$

with the intrinsic viscosity $[\eta]$ in units of $\mathrm{cm}^{3} / \mathrm{g}$. The solvent quality parameter can be extracted from the exponent in the Mark-Houwink relationship $[\eta]=K M_{w}{ }^{(3 v-1)}$ to yield $3 v-1=0.65 \Rightarrow v=0.55$.

Combining this expression with Graessley's expression for coil overlap (Graessley, 1980) we find that for our PEO sample with $c^{*}=0.77 /[\eta] \approx 8.6 \times 10^{-4} \mathrm{~g} / \mathrm{cm}^{3}(0.086 \mathrm{wt} \%)$. The two solutions considered here are thus weakly semi-dilute solutions.

The longest relaxation time of a monodisperse homopolymer in dilute solution is described by the Rouse-Zimm theory (Doi \& Edwards, 1986) and scales with the following parameters:

$$
\lambda \sim \frac{[\eta] \eta_{s} M_{w}}{R T}=\frac{K \eta_{s} M_{w}^{3 v}}{R T}
$$

where the Mark-Houwink relationship has been used in the second equality. The precise prefactor in the Rouse-Zimm theory depends on the solvent quality and the hydrodynamic interaction between different sections of the chain; however it can be approximately evaluated by the following expression (Tirtaatmadja et al. 2004):

$$
\lambda=\frac{1}{\zeta(v)} \frac{[\eta] \eta_{s} M_{w}}{R T},
$$

where $\zeta(3 v)=\sum_{i=1}^{\infty} 1 / i^{3 v}$ represents the summation of the individual modal contributions to the relaxation time. For $v=0.55$ the prefactor is $1 / \zeta(v)=0.463$. The longest relaxation time for the 
PEO solutions utilized in the present study is thus $\lambda=0.34 \mathrm{~ms}$. Christanti \& Walker (2002) use a different prefactor in eq.(3) but report very similar values of the Zimm time constant for PEO solutions of the same molecular weight (but in a more viscous solvent).

This value of the relaxation time represents the value obtained under dilute solution conditions and characteristic of small amplitude deformations so that the individual chains do not interact with each other. However the solutions studied in the present experiment are in fact weakly semidilute solutions and the extensional flow in the neck results in large molecular deformations. Numerous recent studies with dilute solutions of high molecular polymers (Bazilevskii et al. (2001); Stelter et al. (2002), Christanti \& Walker (2001); Tirtaatmadja et al. (2004)) have shown that the characteristic viscoelastic time scale measured in filament thinning or drop break-up experiments is typically larger than the Zimm estimate and is concentrationdependent for concentration values substantially below $c^{*}$. The Zimm time-constant should thus be considered as a lower bound on the polymer time scale that is measured during a capillarythinning and break-up experiment.

\begin{tabular}{|l|c|c|c|c|c|c||c|}
\hline Fluid & $\mathrm{c} / \mathrm{c}^{*}$ & $\sigma[\mathrm{mN} / \mathrm{m}]$ & $\eta_{0}[\mathrm{mPa} . \mathrm{s}]$ & $t_{\text {Rayleigh }}[\mathrm{ms}]$ & $t_{\text {visc }}[\mathrm{ms}]$ & $O h$ & $\lambda[\mathrm{ms}]$ \\
\hline $0.10 \mathrm{wt} \%$ PEO & 1.33 & $61.0 \pm 0.1$ & $2.3 \pm 0.2$ & 20.9 & 1.61 & 0.077 & 1.5 \\
\hline $0.30 \mathrm{wt} \%$ PEO & 4.00 & $60.8 \pm 0.2$ & $8.3 \pm 1.0$ & 20.8 & 5.78 & 0.27 & 4.4 \\
\hline $\begin{array}{l}0.10 \mathrm{wt} \% \text { PEO } \\
\text { in Gly/Water }\end{array}$ & 1.33 & $58.0 \pm 0.1$ & $18.2 \pm 0.5$ & 23.0 & 13.3 & 0.58 & 23.1 \\
\hline
\end{tabular}

Table 1: The physico-chemical and rheological properties of the aqueous polyethylene oxide (PEO) solutions utilized in the present study. The molecular weight of the solute is $M_{w}=2.0 \times 10^{6} \mathrm{~g} / \mathrm{mol}$.

\subsection{Instrumentation}

In the present experiments we have used a Capillary Break-up Extensional Rheometer designed and constructed by Cambridge Polymer Group (www.campoly.com). The diameter of the end plates is $D_{0}=6 \mathrm{~mm}$ and the final axial separation of the plates can be adjusted from $8 \mathrm{~mm}$ to $15 \mathrm{~mm}$. The midpoint diameter is measured using a near infra-red laser diode assembly (Omron ZLA-4) with a beam thickness of $1 \mathrm{~mm}$ at best focus and a line resolution of 
approximately $20 \mu \mathrm{m}$. High resolution digital video is recorded using a Phantom V5.0 high speed camera (at 1000 or 2000 frames/second) with a Nikon $28-70 \mathrm{~mm} \mathrm{f} / 2.8$ lens. Exposure times are $214 \mu$ s per frame. The video is stored digitally using an IEEE1394 firewire link and individual frames are cropped to a size of $512 \times 216$ pixels. The resulting image resolution is $26.8 \mu \mathrm{m} /$ pixel and the overall image magnification is $1.7 \mathrm{X}$.

\subsection{Length-Scales, Time-Scales and Dimensionless Parameters}

The operation of a capillary-thinning rheometer is governed by a number of intrinsic or naturally-occurring length and time scales. It is essential to understand the role of these lengthscales and timescales in controlling the dynamics of the thinning and break-up process. We discuss each of these scales individually below:

The Sample Aspect Ratio; $\Lambda(t)=h(t) / 2 R_{0}$

As indicated in Figure 1, the initial sample is a cylinder with aspect ratio $\Lambda_{0}=h_{0} / 2 R_{0}$. Exploratory numerical simulations for filament stretching rheometry (Harlen, 1996; Yao \& McKinley, 1998) show that optimal aspect ratios are typically in the range $0.5 \leq \Lambda_{0} \leq 1$ in order to minimize the effects of either an initial 'reverse squeeze flow' when the plates are first separated (at low aspect ratios $\Lambda(t) \ll<$ ) or sagging and bulging of the cylindrical sample (at high aspect ratios). The final aspect ratio $\Lambda_{f}=h_{f} / 2 R_{0}$ attained when the plates are separated controls the total Hencky strain that is applied and is discussed further below.

The Capillary Length; $\ell_{c a p}=\sqrt{\sigma / \rho g}$

Surface tension is employed in order to hold the initial fluid sample between the two endplates. It is thus essential that the initial plate separation $\left(h_{0}\right)$ is small enough to support a static liquid bridge. Although the classical Plateau-Rayleigh stability criterion $h_{0} \leq 2 \pi R_{0}$ is well-known (Plateau 1873; Rayleigh 1879), this result only applies in the absence of buoyancy forces. For the case of axial gravity, the situation is more complex and the maximum stable sample size depends on both the fluid volume and the Bond number $B o=\rho g R_{0}^{2} / \sigma$ (Slobozhanin et al. 1993). In order to keep the initial configuration close to cylindrical (with little axial 'sagging') capillary breakup tests typically employ axial separations $h_{0} \leq \ell_{c a p}$ or equivalently $h_{0} / R_{0} \leq 1 / \sqrt{B o}$. For water ( $\rho=1000 \mathrm{~kg} / \mathrm{m}^{3} ; \sigma=0.072 \mathrm{~N} / \mathrm{m}$ ), the capillary length is $\ell_{c a p}=2.7 \mathrm{~mm}$. 


\section{The Viscous Break-up Timescale $\left(t_{v}\right)$}

For a viscous Newtonian fluid, a simple force balance shows that the break-up process proceeds linearly with time (Entov \& Hinch, 1997); and close to break-up the filament profile is found to be self-similar (Papageorgiou, 1995; Eggers, 1997; Chen et al. 2002). These observations can be combined to provide a means of extracting quantitative values for the capillary velocity $v_{\text {cap }}=\sigma / \mu$, or correspondingly the fluid viscosity (if the surface tension is determined independently). Provided gravitational effects are not important (so that $R_{\text {mid }}<\ell_{\text {cap }}$ ), the midpoint radius is given by (McKinley \& Tripathi, 2000):

$$
R_{\text {mid }}(t)=R_{0}-\frac{\sigma}{14.1 \mu} t
$$

The characteristic viscous time scale for the break-up process is thus $t_{v}=14.1 \mu R_{0} / \sigma$.

\section{The Rayleigh Time-scale $\left(t_{R}\right)$}

For a "low viscosity fluid" (to be defined more precisely below), the analysis of Rayleigh for break-up of an inviscid fluid jet is appropriate. The analysis shows that the characteristic time scale for break-up is $t_{R}=\sqrt{ } \rho R_{0}^{3} / \sigma$. For a filament or jet of water with radius 3mm, the Rayleigh time scale is extremely short $t_{R}=0.020 \mathrm{~s}(20 \mathrm{~ms})$. This time scale plays a key role in controlling the operability of filament thinning devices as we show below in the discussion $(\S 4)$.

The question that naturally arises in a capillary-thinning test is what constitutes a "lowviscosity" or, conversely, a high viscosity fluid? This can be answered by comparing the viscous time scale to the Rayleigh time scale. The resultant quantity is a dimensionless number known as the Ohnesorge number

$$
\frac{t_{v}}{t_{R}} \equiv O h=\frac{14.1 \mu}{\sqrt{\rho \sigma R_{0}}}
$$

Note that here we have retained the numerical factor of 14.1 (obtained from the Papageorgiou similarity solution for visco-capillary breakup) in the definition because it is not an $O(1)$ constant. Neglecting this factor leads to quantitative errors in the viscosity obtained from observations of filament thinning (Liang \& Mackley, 1994; Stelter et al. 2000) and an inaccurate estimate for the relative balances of terms controlling with capillary break-up devices. A "lowviscosity fluid" in capillary break-up elongational rheometry thus implies $t_{v}<t_{R}$ (i.e. $O h<1$ ); for aqueous solutions (with $\sigma \approx 0.07 \mathrm{~N} / \mathrm{m} ; R_{0} \approx 3 \mathrm{~mm}$ ) this corresponds to $\mu<0.033$ Pa.s. 
The Opening Time $\left(\delta t_{0}\right)$ and the Imposed Axial Strain $\left(\varepsilon_{f}\right)$

In a torsional step strain experiment, the shear strain is considered theoretically to be applied instantaneously. In reality, the step response of a conventional torsional rheometer is on the order of $25-50 \mathrm{~ms}$ and the torsional displacement is approximately linear with time. By analogy, the axial step strain imposed during a capillary break-up test is typically considered in a theoretical analysis to be imposed instantaneously. In experiments, however the plate separation occurs in a finite time, denoted $\delta t_{0}$. If a servo-system is used to stretch the liquid filament, then this time can be varied and the displacement profile may be linearly or exponentially increasing with time. However, as a result of inertia in the plate \& drive subsystem it typically is constrained to be $\delta t_{0} \geq 0.050 \mathrm{~s}$. Because the filament must not break during the opening process we must require $t_{v} \geq \delta t_{0}$. This criterion sets a stringent lower bound on the Newtonian viscosity that can be tested in a CABER device as we show below.

The initial rapid separation of the endplates also results in the imposition of an initial Hencky strain (a 'pre-strain') of magnitude $\varepsilon_{f}=\ln \left(h_{f} / h_{0}\right)=\ln \left(\Lambda_{f} / \Lambda_{0}\right)$. As a consequence of the no-slip boundary conditions, the deformation of the fluid column is not homogeneous (i.e. the sample does not remain cylindrical); this axial measure of the strain is thus not an accurate measure of the actual Hencky strain experienced by fluid elements near the midplane of the sample. If the initial radius of the sample at time $t_{0}$ is $R_{0}$ and the midpoint radius of the filament at time $t_{1}=t_{0}+\delta t_{0}$ is denoted $R_{1}$, then the true Hencky pre-strain imposed during the stretching process is $\varepsilon_{1}=2 \ln \left(R_{0} / R_{1}\right)$. It is not possible to predict this final radius $R_{1}$ without choosing a constitutive model for the fluid; however, for many test samples (with $t_{v} \gg \delta t_{0}$ ), the midpoint radius of the sample at the cessation of the stretching is given by the lubrication solution for a viscous Newtonian fluid (Spiegelberg et al. 1996):

$$
R_{1} \approx R_{0}\left(L_{f} / L_{0}\right)^{-3 / 4}
$$

\section{The Polymer Relaxation Time $(\lambda)$}

If the test fluid in a capillary thinning test is a polymer solution, then non-Newtonian elastic stresses grow during the transient elongational stretching process. Ultimately these extensional stresses grow large enough to overwhelm the viscous stress in the neck. An elastocapillary force balance then predicts that the filament radius decays exponentially in time 


$$
\frac{R_{\text {mid }}(t)}{R_{0}}=\left(\frac{G R_{0}}{2 \sigma}\right)^{1 / 3} \exp [-t / 3 \lambda] .
$$

The additional factor of $2^{-1 / 3}$ in the prefactor of eq. (7) is missing in the original theory (Entov \& Hinch, 1997) due to a simplifying approximation made in deriving the governing equation (Clasen et al. 2004). This simplification however does not change the exponential factor that is used to measure the characteristic time constant of the polymeric liquid. This relationship has been utilized to determine the relaxation time for many different polymeric solutions over a range of concentrations and molecular weights (Basilevskii et al. (1990); Liang \& Mackley (1994); Basilevskii et al. (1997); Anna \& McKinley (2000); Stelter et al. (2000)).

Note that although this time constant is referred to as a 'relaxation time' - because it is the same time constant that is associated with stress relaxation following cessation of steady shear - in a capillary-thinning experiment, the stress is not relaxing per se. In fact the tensile stress diverges as the radius decays to zero. The time constant obtained from a CABER test is thus more correctly referred to as the "characteristic time scale for viscoelastic stress growth in a uniaxial elongational flow'. This is, of course, precisely the time constant of interest in commercial operations concerned with drop break-up, spraying, mold-filling, etc.

For low viscosity systems, however, this exponential decay becomes increasingly difficult to observe due to the formation of well-known beads-on-a-string morphology (Goldin et al. 1969; Li \& Fontelos, 2003). The elastic stresses in the necking filament grow on the characteristic scale $\lambda$ and must grow sufficiently large to resist the growth of free-surface perturbations, which evolve on the Rayleigh time scale, $t_{R}$. In the same manner that comparison of the viscous and Rayleigh time-scales resulted in a dimensionless group (the Ohnesorge number) so too does comparison of the polymer time-scale and the Rayleigh time scale. This dimensionless ratio may truly be thought of as a Deborah number (Bird et al. 1987) because it compares the magnitude of the polymeric time scale with the flow time scale for the necking process in a low viscosity fluid:

$$
D e \equiv \frac{\lambda}{t_{R}}=\frac{\lambda}{\sqrt{\rho R_{0}^{3} / \sigma}} .
$$

Note however that because the necking filament is not forced by an external deformation, it selfselects the characteristic time scale for the necking process. This Deborah number is thus an 'intrinsic quantity' that cannot be affected by the rheologist; except in so far as changes in the 
concentration and molecular weight of the test fluid change the characteristic time constant of the fluid.

As we have shown above the Rayleigh timescale is short and thus results in rapid stretching in the fluid filament with strain rates $\dot{\varepsilon} \sim t_{R}{ }^{-1} \geq 50 \mathrm{~s}^{-1}$. It should thus be possible to test low viscosity fluids with small relaxation time constants. The question is how small? In the experiments described below, we seek to find for what range of Deborah numbers it is possible to use Capillary Break-up Extensional rheometry to determine the relaxation time of low viscosity fluids.

\section{$3 \quad$ Results}

\subsection{Beads on a String and Inertio-Capillary Oscillations}

In figure 2 we present a sequence of digital video images that demonstrate the time evolution in the filament profile for the $0.10 \mathrm{wt} \%$ PEO solution; corresponding to a very low Deborah number, $D e=0.074$. In all of the experiments presented in this paper we define the time origin to be the instant at which axial stretching ceases, so that $t=t_{l a b}-\delta t_{0}$. The first image at time $t=-0.05 \mathrm{~s}$ thus corresponds to the initial configuration of the liquid bridge with $\Lambda_{0}=3 \mathrm{~mm} / 6 \mathrm{~mm}=0.5$. We also report the total time for the break-up event to occur as determined from analysis of the digital video sequence; with the present optical and lighting configuration the uncertainty in determining the break-up time is approximately $\pm 0.005 \mathrm{~s}$. For consistency we then show a sequence of five images that are evenly spaced throughout the break-up process. The horizontal broken lines indicate the approximate width of the laser light sheet that is projected by the laser micrometer.

From Figure 2, it is clear that initially, during the first $25 \mathrm{~ms}$ of the axial stretching phase, the filament profile remains axially symmetric and a neck forms near the midplane as expected. However, this axial symmetry is not maintained at the end of the stretching sequence and a local defect or 'ligament' forms near the lower plate. Following the cessation of stretching, the filament rapidly evolves into a characteristic beads-on-a-string structure with a primary droplet and several smaller 'satellite droplets'. The hemispherical blobs attached to each end plate oscillate with a characteristic time scale that is proportional to the Rayleigh time constant, $t_{R}=22$ ms. 
The strong asymmetry in the axial curvature that can be observed in the thin ligament which develops at $t=0$ is a hallmark of an inertially-dominated break-up process (Eggers, 1997); the viscous time-scale is only $t_{\mathrm{v}}=1.6 \mathrm{~ms}$ for this low viscosity fluid, hence we find $O h<<1$ and the absence of an axially-uniform filament near the midplane suggests additionally that $D e<<1$. It is thus not easy to use such experiments to extract the viscoelastic time constant of the fluid. Elastic stresses only become important on very small length scales and very short time scales when the stretching rate associated with Rayleigh break-up becomes sufficiently large. From eq. (8) we may estimate this length scale by equating the polymeric and inertial (Rayleigh) time scales (i.e. by setting $D e \sim 1$ ) to find $R_{\text {ligament }} \sim\left(\lambda^{2} \sigma / \rho\right)^{1 / 3}$. Conversely, the effective relaxation time may be estimated from observing the size of the viscoelastic ligament that initially forms when elastic effects first become important. From Figure $2(\mathrm{c})$ we estimate $R_{\text {ligament }} \approx 0.3 \mathrm{~mm}$, thus indicating that $\lambda=\left(\rho R_{\text {ligament }}^{3} / \sigma\right)^{1 / 2} \approx 1 \mathrm{~ms}$. Such a measurement is clearly imprecise; but serves to provide an a priori estimate that can be used to compare with better measurements we make below.

A second example of difficulties that can be encountered with CABER measurements is shown in Figure 3 for the $0.1 \mathrm{wt} \%$ PEO solution in water/glycerol. The increased viscosity of the fluid delays the break-up event substantially and the increased relaxation time of the polymer leads to the formation of an axially uniform fluid ligament as desired. However, inertial oscillations of the hemispherical droplets attached to each endplate still occur. The low aspect ratio of the selected test configuration $\left(h_{f}=8.46 \mathrm{~mm} ; \Lambda_{f}=1.41\right)$ results in these oscillations intruding into the observation plane of the laser micrometer. The period of these fluctuations may be estimated from the theory for oscillations of an inviscid liquid drop (Chandrasekhar, 1962). The fundamental mode has a period $t_{o s c} \equiv 2 \pi / \omega_{\text {osc }}=(\pi / \sqrt{2}) t_{R}$. For the $0.1 \mathrm{wt} \%$ PEO solution this gives $t_{\text {osc }} \approx 46 \mathrm{~ms}$ in good agreement with the experimental observations.

The consequences can be seen in Figure 4(a) which shows the evolution in the midpoint diameter $D_{\text {mid }}(t)=2 R_{\text {mid }}(t)$. The oscillations can be clearly seen in the data for the $0.10 \mathrm{wt} \%$ glycerin/water solution; however the exponential decay in the radius at long times can still be clearly discerned; and the data can be fitted to a decaying exponential of the form given by equation (7). The value of the characteristic time constant for each measurement is shown on the 
figure. The lower viscosity $0.10 \mathrm{wt} \%$ and $0.30 \mathrm{wt} \%$ solutions break very rapidly; typically within the period of a single oscillation.

The effects of varying the imposed stretch, i.e. the final aspect ratio $\Lambda_{f}=h_{f} / 2 R_{0}$, on the evolution of the midplane diameter is shown in Figure 4(b). At the highest aspect ratio $\left(\Lambda_{f}=2\right)$, corresponding to the high-speed digital images shown in Figure 2, the measurements do not show exponential thinning behavior as a consequence of the large liquid droplet passing through the measuring plane. As the aspect ratio is decreased, the data begins to approximate exponential behavior and regression of eq. (7) to the data results in reasonable estimates of the relaxation time.

\subsection{Sample Size and Volume}

As we noted above in $\S I$ II the initial sample configuration can play an important role in ensuring that capillary break-up rheometry yields reliable and successful results. By analogy, in conventional torsional rheometry it is key to ensure that the cone angle of the fixture is sufficiently small or that the gap separation for a parallel plate fixture is in a specified range. In Figures 5-7 we show the consequences of varying the initial sample gap height, as compared to the capillary length $\ell_{c a p}=\sqrt{\sigma / \rho g}$. In each test we use the $0.30 \mathrm{wt} \%$ PEO solution and a fixed final aspect ratio of $\Lambda=1.6$; corresponding to a final stretching length $h_{f}=1.61\left(2 R_{0}\right)=9.7 \mathrm{~mm}$.

If $h_{0} / \ell_{\text {cap }}<1$ then the interfacial force arising from surface tension is capable of supporting the liquid bridge against the sagging induced by the gravitational body force; consequently the initial sample is approximately cylindrical and the initial deformation results in a top-bottom symmetric deformation and the formation of an axially-uniform ligament at $t=0$ when deformation ceases. However, if the initial gap is larger, as shown in Figure 6 (corresponding here to $\left.h_{0}=3 \mathrm{~mm}\right)$ and exceeds the capillary length scale $\left(h_{0} / \ell_{\text {cap }}=1.19\right)$, then asymmetric effects arising from gravitational drainage become increasingly important. Even under rest conditions (as shown by the first image in Figure 6), gravitational effects result in a detectable bulging in the lower half of the liquid bridge; as predicted numerically (Slobozhanin et al. 1992). This asymmetry is amplified during the 'strike' or gap-opening process as indicated in the $2^{\text {nd }}$ and $3^{\text {rd }}$ frames. However as viscoelastic stresses in the neck region grow and a thin elastic thread develops, the process stabilizes and exponential filament thinning occurs once again. In Figure 7 we show an even more pronounced effect when the initial gap is $4 \mathrm{~mm}$ 
(corresponding to $h_{0} / \ell_{\text {cap }}=1.58$ ). The asymmetry of the initial condition and the extra fluid volume (corresponding to a volume of $V \approx \pi R_{0}^{2} h_{0}=113 \mu \mathrm{l}$; i.e. twice the fluid volume in Figure 5 ) is sufficient to initialize the formation of a 'bead' or droplet near the middle of the filament at $t=25 \mathrm{~ms}$, which subsequently drains into the lower reservoir. A distinct uniform axial thread only develops for times greater than $t \geq 0.3 t_{\text {event }} \approx 0.04 \mathrm{~s}$. This severely limits the useful range of measurements.

The measured midpoint diameters for the conditions in Figures 5 - 7 are shown in Figure 8. The progressive drainage of the primary droplet through the measuring plane of the laser micrometer can be clearly seen in the data for $h_{0}=4 \mathrm{~mm}$. Although an exponential regime (corresponding to elasto-capillary thinning with approximately constant slope of the form given by eq. (7)) can be seen for the intermediate separation $\left(h_{0}=3 \mathrm{~mm}\right)$, the perturbing effects of axial drainage result in fluctuations in the diameter profile and an under-prediction in the longest relaxation time. The smallest initial gap setting $\left(h_{0}=2 \mathrm{~mm}\right)$, however, results in steady exponential decay over a time period of approximately $\Delta t=40 \mathrm{~ms}$; corresponding to $\Delta t / 3 \lambda \approx 1.8$ and, consequently from eq.(7) a diameter decrease of more than a factor of 6 . This is of a sufficiently wide range to satisfactorily regress to the equation.

One important feature to note from a careful comparison of Figures 7 and 8 is the difference in spatial resolution offered by the digital imaging system; the laser micrometer has a calibrated spatial resolution of ca. $20 \mu \mathrm{m}$ (Anna \& McKinley, 2001) which is reached after a time interval of approximately $\Delta t \approx 50 \mathrm{~ms}$; hence $\Delta t / t_{\text {event }} \approx 50 / 125=0.4$. By contrast, a thin elastic ligament can still be visually discerned for another $50 \mathrm{~ms}$. The performance of future Capillary Break-up Extensional Rheometers may thus be enhanced by employing laser micrometers with higher spatial resolution or using analog/digital converters with 16bit or 20bit resolution. Such devices however typically become increasingly bulky and expensive.

\subsection{The Role of Fluid Viscosity and Aspect Ratio}

As we noted in $\S 2.1$, the longest relaxation time and also the zero-shear rate viscosity of a dilute polymer solution both vary with the viscosity of the background Newtonian solvent and also with the concentration of the polymer in solution. The characteristic viscous and elastic time scales associated with the break-up process also increase and so do the dimensionless parameters

$O h$ and $D e$. Inertial effects thus become progressively less important and capillary break-up 
experiments become concomitantly easier. An example is shown in Figure 9 for the $0.1 \mathrm{wt} \%$ PEO solution in glycerol/water at a high aspect ratio $\left(\Lambda_{f}=2.0\right)$. The equivalent process in a purely aqueous solvent has already been shown in Figure 2 and resulted in a beads-on-string structure that corrupted CABER experiments. However, by increasing the background solvent viscosity this break-up process is substantially retarded (the total time for break-up increases from $50 \mathrm{~ms}$ to over $400 \mathrm{~ms}$ ) and a uniform fluid filament is formed between the upper and lower plates. The corresponding midpoint diameter measurements for each of the test fluids (in this case with a reduced aspect ratio of $\Lambda_{f}=1.6$ and an initial gap of $h_{0}=3 \mathrm{~mm}$ ) are shown in Figure 10(a). For the $0.10 \mathrm{wt} \%$ PEO solution in Glycerin/Water a statistically significant deviation from a pure exponential decay can be observed for $t \geq 0.18 \mathrm{~s}$. This corresponds to the onset of finite extensibility effects associated with the PEO molecules in the stretched elastic ligament attaining full extension (Entov \& Hinch, 1997). In this final stage of break-up, numerical simulations with both the FENE-P and Giesekus models show that the filament radius decreases linearly with time (Fontelos \& Li, 2004).

Finally, our results for the measured relaxation times of the three test fluids are summarized in Figure 10(b). Each point represents the average of at least three tests under the specified experimental conditions. No data could be obtained with the $0.1 \mathrm{wt} \% \mathrm{PEO} /$ water solution at aspect ratios $\Lambda \geq 1.8$ due to the inertio-capillary break-up and beads-on-a-string morphology shown in Figure 2. It can be noted that the measured relaxation times vary with aspect ratio very weakly. This is reassuring for a rheometric device and indicates that relaxation times as small as $\lambda \approx 1 \mathrm{~ms}$ can successfully be measured using capillary thinning and break-up experiments. Average values of the measured relaxation times are tabulated in the final column of Table 1.

\section{Discussion \& Conclusions}

In this paper we have performed capillary break-up extensional rheometry (CABER) experiments on a number of semi-dilute polymer solutions of varying viscosities using cylindrical samples of varying initial size and imposed stretches of different axial extent leading to various imposed axial strains. High speed digital imaging shows that changes in these parameters may change the dynamics of the filament thinning and break-up process for each fluid substantially. 
By considering the natural length scales and time scales that govern these dynamics, we have been able to develop a number of dimensionless parameters that control the successful operability of such devices as extensional rheometers; the most important being the Ohnesorge number, a natural or 'intrinsic' Deborah number and the Bond number. These constraints can perhaps be most naturally represented in the form of an 'operability diagram' such as the one sketched in Figure 11; in which we select the dimensional parameters corresponding to the zeroshear-rate viscosity $\left(\eta_{0}\right)$ of the solution and the characteristic relaxation time $(\lambda)$ as the abscissa and ordinate axes respectively. A more general version of the same diagram could be shown in terms of the Ohnesorge and Deborah numbers.

For Newtonian fluids (corresponding to $\lambda=0$ ) we require, at a minimum, that $t_{v} \geq t_{R}$ (or $O h \geq 1$ ) in order to observe the effects of fluid viscosity on the local necking and break-up. As we discussed in $\S 2.3$ for the present configuration this gives a lower bound on the measurable viscosity of $33 \mathrm{mPa}$.s. However, the device also takes a finite time (which we denote $\delta t_{0}$ ) to impart the initial axial deformation to the sample. An additional constraint is thus $t_{v} \geq \delta t_{0}$ or

$$
\mu \geq \frac{\sigma}{14.1 R_{0}} \delta t_{0}
$$

For a prototypical Newtonian fluid with $\sigma \approx 0.060 \mathrm{~N} / \mathrm{m}$, a plate size of $R_{0}=3 \mathrm{~mm}$ and an opening time of $\delta t_{0}=50 \mathrm{~ms}$ we find $\mu \geq 0.071$ Pa.s. This defines the intersection of the operability boundary with the abscissa. Increasing the displacement rate of the linear motor in order to reduce the opening time would enable somewhat lower viscosity fluids to be tested; however the natural Rayleigh time scale for break-up of a Newtonian fluid thread will ultimately limit the range of viscosities that can be successfully tested.

The dilute polymer solutions tested in the present study obviously have viscosities significantly less than this value, and viscoelasticity further stabilizes the filament against breakup. The simplest estimate for the range of relaxation times that can be measured is to require $D e \geq 1$ or equivalently $\lambda \geq t_{R}=\sqrt{\rho R_{0}^{3} / \sigma} \approx 20 \mathrm{~ms}$. However this estimate is based on an elastocapillary balance in a thread of radius $R_{0}$. In reality we are able to resolve thinning threads of substantially smaller spatial scale. Closer analysis of the digital video from which the images in Figure 2 are taken (specifically, the frames from between times $t=-25 \mathrm{~ms}$ and $0 \mathrm{~ms}$ which are not presented here) shows that a neck first forms at $t=-5 \mathrm{~ms}$, when the thread diameter at the neck is approximately $200 \mu \mathrm{m}$; the minimum resolvable viscoelastic relaxation time should thus be $\lambda>\sqrt{\left(10^{3}\right)\left(2 \times 10^{-4}\right)^{3} /(0.06)} \approx 0.4 \mathrm{~ms}$. 
However, just as in the above arguments regarding the minimum measurable Newtonian viscosity, the capabilities of the instrumentation also play a role and may serve to further constrain the measurable range of material parameters. More specifically, the minimum measurable radius, the total imposed stretch and the sampling rate will all impact the extent to which a smoothly decaying exponential of the form required by eq. (7) can be resolved. In the present experiments we have sampled the analog diameter signal from the laser micrometer at a rate of $1000 \mathrm{~Hz}\left(\delta t_{s}=0.001 \mathrm{~s}\right)$, and the minimum radius that can be reliably detected by the laser micrometer is $R_{\min } \approx 20 \mu \mathrm{m}$. If we require that, as an absolute minimum, we monitor the elastocapillary thinning process long enough to obtain 5 points that can be fitted to an exponential curve, then the measured radius data must span the range $R_{\min } \leq R_{\text {mid }}(t) \leq R_{\min } e^{+5 \delta t_{s} / 3 \lambda}$. However the radius of the neck at the cessation of the imposed stretching $(t=0)$ is given (at least approximately) by eq. (6). Combining these expressions we thus require that

$$
R_{\min } e^{5 \delta t_{s} / 3 \lambda} \leq R_{1}=R_{0}\left(\Lambda_{f}\right)^{-3 / 4}
$$

Rearranging this expression gives:

$$
\lambda \geq \frac{5 \delta t_{s}}{\ln \left[R_{0} \Lambda_{f}^{-3 / 4} / R_{\min }\right]}
$$

For an axial stretch of $\Lambda_{f}=1.6$, a sampling time of $1 \mathrm{~ms}$, and a minimum detectable radius of $20 \mu \mathrm{m}$ we obtain a revised estimate of the minimum measurable relaxation time $\lambda \geq 0.34 \mathrm{~ms}$, which is in agreement with our present observations. In reality, eq.(6) is an overestimate of the neck radius $\left(R_{1}\right)$ at the cessation of the stretching phase, since the lubrication theory from which it is derived implicitly assumes viscous effects are fully developed throughout the axial stretching process. The data in Figure 4 show that, in general, for low viscosity fluids the exponential necking phase starts at a somewhat lower value of the measured radius. This will increase the lower bound given by eq.(9); however the weak logarithmic dependence of this expression on the precise value of the radius makes such corrections small.

This estimate of the minimum viscoelastic time scale denotes the limiting bound of successful operation for a very low viscosity (i.e. an almost inviscid) elastic fluid; corresponding to the ordinate axis $(O h \rightarrow 0)$ of Figure 11. The shape and precise locus of the operability boundary within the two-dimensional interior of this parameter space will depend on all three time scales (viscous, elastic and inertial) and also on the initial sample size $\left(h_{0} / \ell_{\text {cap }}\right)$ and the 
total axial stretch $\left(\Lambda_{f}\right)$ imposed. It thus needs to be studied in detail through numerical simulations. However, our experiments indicate that it is possible, through careful selection of both the initial gap $\left(h_{0}\right)$ and the final strike distance $\left(h_{f}\right)$, to successfully measure relaxation times as small as $1 \mathrm{~ms}$ for low viscosity elastic fluids with zero-shear rate viscosities as small as 3 mPa.s.

A final practical use of an operability diagram such as the one sketched in Figure 11 is that it enables the formulation chemist and rheologist to understand the consequences of changes in the formulation of a given polymeric fluid. The changes in the zero-shear-rate viscosity and longest relaxation time that are expected from dilute solution theory and formulae such as eq. (3) are indicated by the arrows. Increases in the solvent quality and molecular weight of the solute lead to large changes in the relaxation time, but small changes in the overall solution viscosity (at least under dilute solution conditions). By contrast, increasing the concentration of dissolved polymer into the semi-dilute and concentrated regimes leads to large increases in both the zeroshear-rate viscosity and the longest relaxation time. It should be noted that the dynamics of the break-up process can change again at very high concentrations or molecular weights when the solutions enter the entangled regime (corresponding to $c M_{w} \geq \rho M_{e}$, where $M_{e}$ is the entanglement molecular weight of the melt). Although capillary thinning and break-up experiments can still be successfully performed, the dimensionless filament lifetime $t_{\text {event }} / \lambda$ (as expressed in multiples of the characteristic relaxation time) may actually decrease from the values observed in the present experiments due to chain disentanglement effects (Bhattacharjee et al. 2003); i.e. a concentrated polymer solution may actually be less extensible than the corresponding dilute solution. Capillary thinning and break-up experiments of the type described in this article enable such effects to be systematically probed. 


\section{Bibliography}

Amarouchene, Y., Bonn, D., Meunier, J. and Kellay, H., Inhibition of the Finite Time Singularity during Droplet Fission of a Polymeric Fluid, Phys. Rev. Lett., 86(16), (2001), 3558-2562.

Anna, S.L. and McKinley, G.H., Elasto-capillary Thinning and Breakup of Model Elastic Liquids, J. Rheol., 45(1), (2001), 115-138.

Bazilevskii, A.V., Entov, V.M., Lerner, M.M. and Rozhkov, A.N., Failure of Polymer Solution Filaments, Polymer Science Ser. A (translated from Vysokomolekulyarnye Soedineniya Ser. A pp. 474-482), 39(3), (1997), 316-324.

Bazilevskii, A.V., Entov, V.M. and Rozhkov, A.N., Failure of an Oldroyd Liquid Bridge as a Method for Testing the Rheological Properties of Polymer Solutions, Polymer Science Ser. A (translated from Vysokomolekulyarnye Soedineniya Ser. A pp. 474-482), 43(7), (2001), 11611172 .

Bazilevsky, A.V., Entov, V.M. and Rozhkov, A.N., "Liquid Filament Microrheometer and Some of its Applications", Third European Rheology Conference, D. R. Oliver (ed.), Elsevier Applied Science, 1990, 41-43.

Bhattacharjee, P.K., Nguyen, D.A., McKinley, G.H. and Sridhar, T., Extensional Stress Growth and Stress Relaxation in Entangled Polymer Solutions, J. Rheol., 47(1), (2003), 269-290.

Bird, R.B., Armstrong, R.C. and Hassager, O., Dynamics of Polymeric Liquids. Volume 1: Fluid Mechanics, 2nd Edition, Wiley Interscience, New York, 1987.

Brandrup, H. and Immergut, E.H., Polymer Handbook, Wiley, New York, 1997.

Chandrasekhar, S., Hydrodynamic and Hydromagnetic Stability, Dover, New York, 1961, 475477.

Chen, A.U., Notz, P.K. and Basaran, O.A., Computational and Experimental Analysis of PinchOff and Scaling, Phys. Rev. Lett., 88(17), (2002), 174501-4.

Christanti, Y. and Walker, L., Surface tension driven jet break up of strain-hardening polymer solutions, J. Non-Newt. Fluid Mech., 100(1-3), (2001), 9-26.

Christanti, Y.M. and Walker, L., Effect of Fluid Relaxation Time on Jet Breakup due to a Forced Disturbance of Polymer Solutions, J. Rheol., 46(3), (2002), 733-739.

Clasen, C., Verani, M., Plog, J.P., McKinley, G.H. and Kulicke, W.-M., Effects of Polymer Concentration and Molecular Weight on the Dynamics of Visco-Elasto-Capillary Breakup, Proc. XIVth Int. Cong. Rheol., Seoul, (S. Korea), August 2004 
Cooper-White, J.J., Fagan, J.E., Tirtaatmadja, V., Lester, D.R. and Boger, D.V., Drop Formation Dynamics of Constant Low Viscosity Elastic Fluids, J. Non-Newt. Fluid Mech., 106, (2002), 2959.

Doi, M. and Edwards, S.F., The Theory of Polymer Dynamics, OUP, Oxford, 1986.

Dontula, P., Pasquali, M., Scriven, L.E. and Macosko, C.W., Can Extensional Viscosity be Measured with Opposed-Nozzle Devices, Rheol. Acta, 36, (1997), 429-448.

Dontula, P., Pasquali, M., Scriven, L.E. and Macosko, C.W., Model Elastic Liquids with WaterSoluble Polymers, AIChE J., 44(6), (1998), 1247-1256.

Eggers, J., Nonlinear Dynamics and Breakup of Free-Surface Flows, Rev. Mod. Phys., 69(3), (1997), 865-929.

Entov, V.M. and Hinch, E.J., Effect of a Spectrum of Relaxation Times on the Capillary Thinning of a Filament of Elastic Liquid, J. Non-Newt. Fluid Mech., 72(1), (1997), 31-54.

Fontelos, M.A. and Li, J., On the Evolution and Rupture of Filaments in Giesekus and FENE models, J. Non-Newt. Fluid Mech., 118(1), (2004), 1-16.

Fuller, G.G., Cathey, C.A., Hubbard, B. and Zebrowski, B.E., Extensional Viscosity Measurements for Low Viscosity Fluids, J. Rheol., 31(3), (1987), 235-249.

Goldin, M., Yerushalmi, H., Pfeffer, R. and Shinnar, R., Breakup of a Laminar Capillary Jet of a Viscoelastic Fluid, J. Fluid Mech., 38(4), (1969), 689-711.

Graessley, W.W., Polymer Chain Dimensions and the Dependence of Viscoelastic Properties on Concentration, Molecular Weight and Solvent Power, Polymer, 21, (1980), 258-262.

Harlen, O.G., Simulation of the Filament Stretching Rheometer, (1996), presentation at the Isaac Newton Institute during Dynamics of Complex Fluids (DCF) program.

Harrison, G.M. and Boger, D.V., Well-Characterized Low Viscosity Elastic Liquids, Appl. Rheol., 10(4: July/Aug), (2000), 166-177.

Hermansky, C.G. and Boger, D.V., Opposing-Jet Viscometry of Fluids with Viscosity Approaching That of Water, J. Non-Newt. Fluid Mech., 56, (1995), 1-14.

Kolte, M.I. and Szabo, P., Capillary Thinning of Polymeric Filaments, J. Rheology, 43(3), (1999), 609-626.

Li, J. and Fontelos, M.A., Drop Dynamics on the Beads-on-String Structure for Viscoelastic Jets: A Numerical Study, Phys. Fluids, 15(4), (2003), 922-937.

Liang, R.F. and Mackley, M.R., Rheological Characterization of the Time and Strain 
Dependence for Polyisobutylene Solutions, J. Non-Newtonian Fluid Mech., 52, (1994), 387-405.

McKinley, G.H., "A Decade of Filament Stretching Rheometry", Proceedings of the. XIIIth Int. Cong. Rheology, Vol.1, D. M. Binding, N. E. Hudson, J. Mewis, J.-M. Piau, C. J. S. Petrie, P. Townsend, M. H. Wagner and K. Walters (ed.), British Society of Rheology, Cambridge (UK), 2000, 15-22.

McKinley, G.H., "Visco-Elasto-Capillary Thinning and Breakup of Complex Fluids", Rheology Reviews, Vol.3, D.M.Binding and K.Walters (ed.), 2005, to appear.

McKinley, G.H. and Tripathi, A., How to Extract the Newtonian Viscosity from Capillary Breakup Measurements in a Filament Rheometer, J. Rheol., 44(3), (2000), 653-671.

Neal, G., Braithwaite, G.J.C., The Use of Capillary Breakup Rheometry to Determine the Concentration Dependence of Relaxation Time, 75th Annual Meeting, SOR, Pittsburgh, 2003, Paper ER1.

Ng, S.L., Mum, R.P., Boger, D.V. and James, D.F., Extensional Viscosity Measurements of Dilute Polymer Solutions of Various Polymers, J. Non-Newt. Fluid Mech., 65, (1996), 291-298.

Papageorgiou, D.T., On the Breakup of Viscous Liquid Threads, Phys. Fluids, 7(7), (1995), 1529-1544.

Plateau, J.A.F., "Experimental and Theoretical Researches on the Figures of Equilibrium of a Liquid Mass Withdrawn from the Action of Gravity", Ann Rep. Smithsonian Institution, 1863, 207-285.

Rayleigh, L., On the Instability of Jets, Proc. Lund. Math. Soc., 10, (1879), 4-13.

Renardy, M., "Self-Similar Breakup of Non-Newtonian Fluid Jets", Rheology Reviews, Vol.2, D.M.Binding and K.Walters (ed.), 2004, 171-196.

Schümmer, P. and Tebel, K.H., A New Elongational Rheometer for Polymer Solutions, J. NonNewt. Fluid Mech., 12, (1983), 331-347.

Slobozhanin, L.A. and Perales, J.M., Stability of Liquid Bridges between Equal Disks in an Axial Gravity Field, Phys. Fluids A, 5(6), (1993), 1305-1314.

Spiegelberg, S.H., Ables, D.C. and McKinley, G.H., The Role of End-Effects on Measurements of Extensional Viscosity in Viscoelastic Polymer Solutions With a Filament Stretching Rheometer, J. Non-Newtonian Fluid Mech., 64(2-3), (1996), 229-267.

Stelter, M., Brenn, G., Yarin, A.L., Singh, R.P. and Durst, F., Validation and Application of a Novel Elongational Device for Polymer Solutions, J. Rheol., 44(3), (2000), 595-616.

Stelter, M., Brenn, G., Yarin, A.L., Singh, R.P. and Durst, F., Investigation of the Elongational 
Behavior of Polymer Solutions by Means of an Elongational Rheometer, J. Rheol., 46(2), (2002), 507-527.

Tirtaatmadja, V., McKinley, G.H. and Boger, D.V., Drop Formation and Breakup of Low Viscosity Elastic Fluids: Effects of Concentration and Molecular Weight, Phys. Fluids, (2004), submitted.

Tripathi, A., Whittingstall, P. and McKinley, G.H., Using Filament Stretching Rheometry to Predict Strand Formation and "Processability" in Adhesives and Other Non-Newtonian Fluids, Rheol. Acta, 39, (2000), 321-337.

Willenbacher, N., Elongational Viscosity of Aqueous Thickener Solutions from Capillary Breakup Elongational Rheometry (CaBER), Proc. XIVth Int. Cong. Rheol., Seoul, (S. Korea), August 2004

Yao, M. and McKinley, G.H., Numerical Simulation of Extensional Deformations of Viscoelastic Liquid Bridges in Filament Stretching Devices, J. Non-Newtonian Fluid Mech., 74((1-3)), (1998), 47-88. 


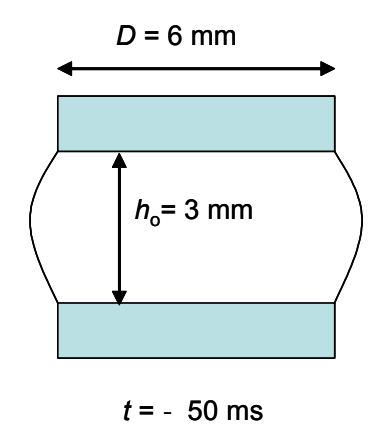

(a)

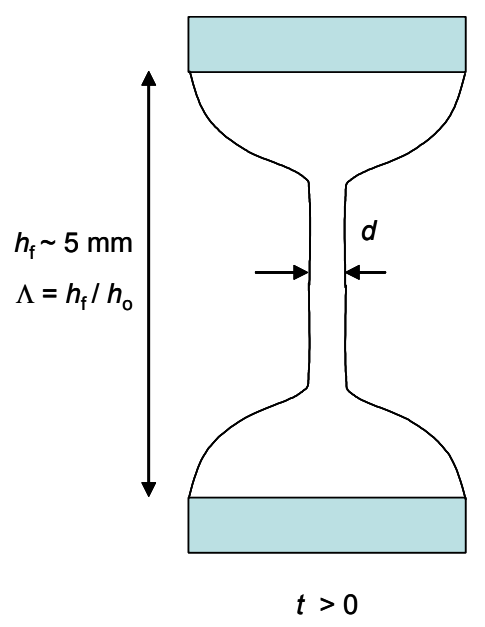

(b)

Figure 1. Schematic of the Capillary Breakup Extensional Rheometer (CaBER) geometry containing a fluid sample (a) at rest and (b) undergoing filament thinning for $t>0$

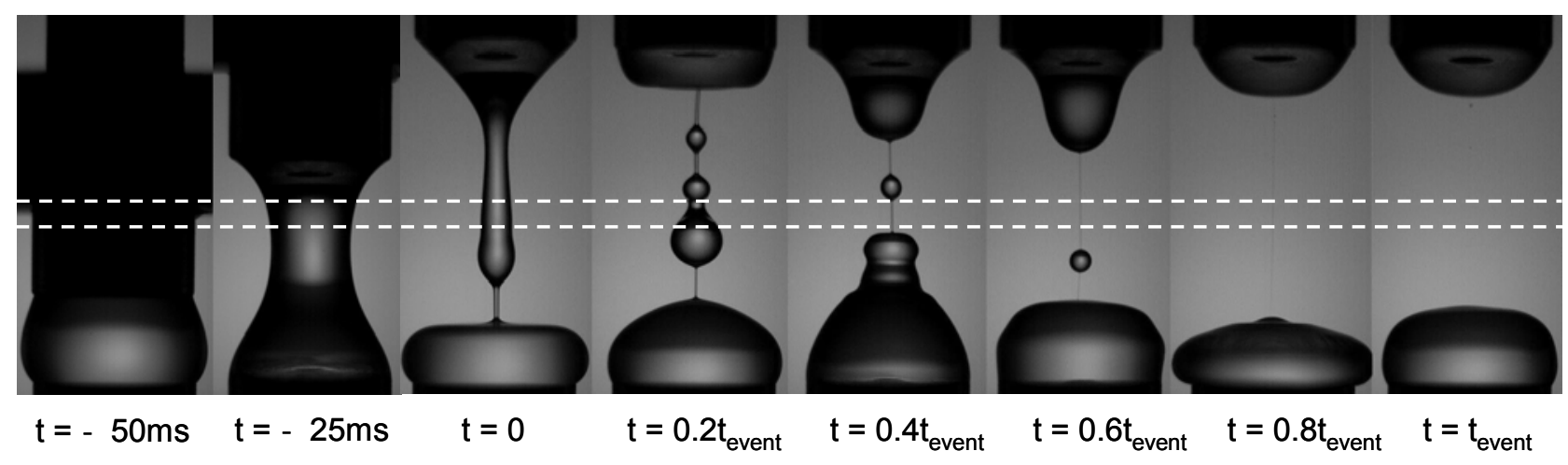

Figure 2. Formation of a beads-on-string and droplet in the $0.1 \%$ PEO fluid filament for $\Lambda=2.0$ and $h_{\mathrm{o}}=3 \mathrm{~mm}$, in which $t_{\text {event }}=50 \mathrm{~ms}$ 


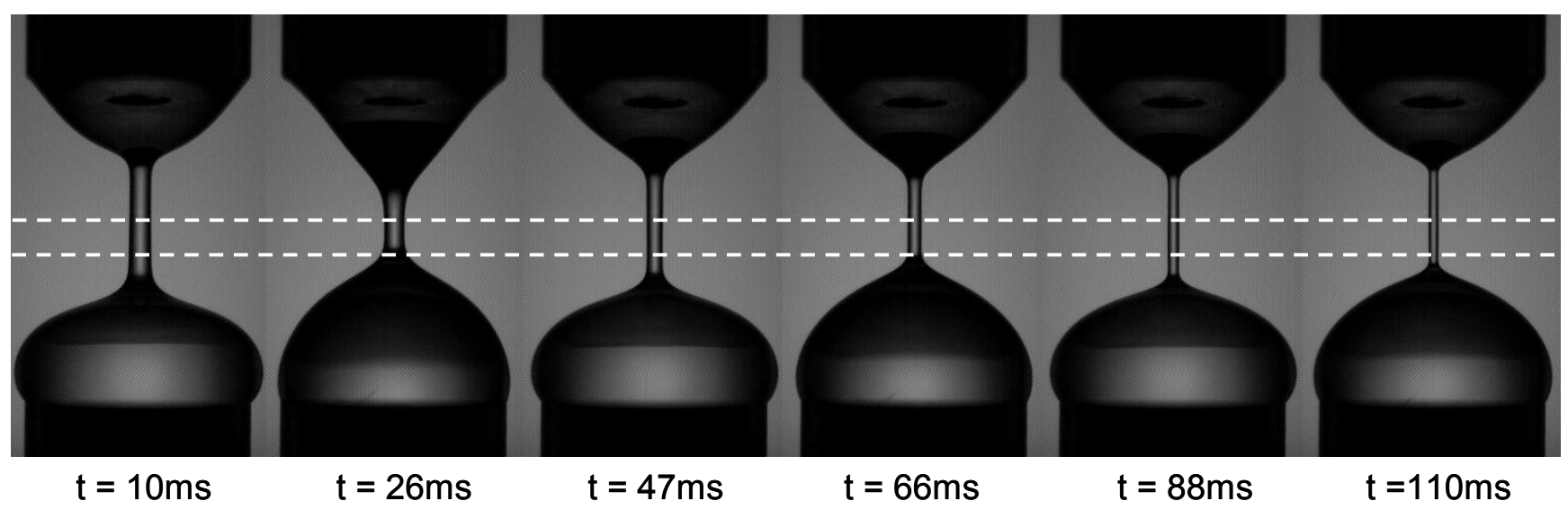

Figure 3. Periodic growth and thinning of the filament diameter due to the inertial oscillation of the fluid end-drops seen in the $0.1 \% \mathrm{PEO} /$ glycerol solution at early times, for $\Lambda=1.41$ and $h_{0}=3 \mathrm{~mm}$.
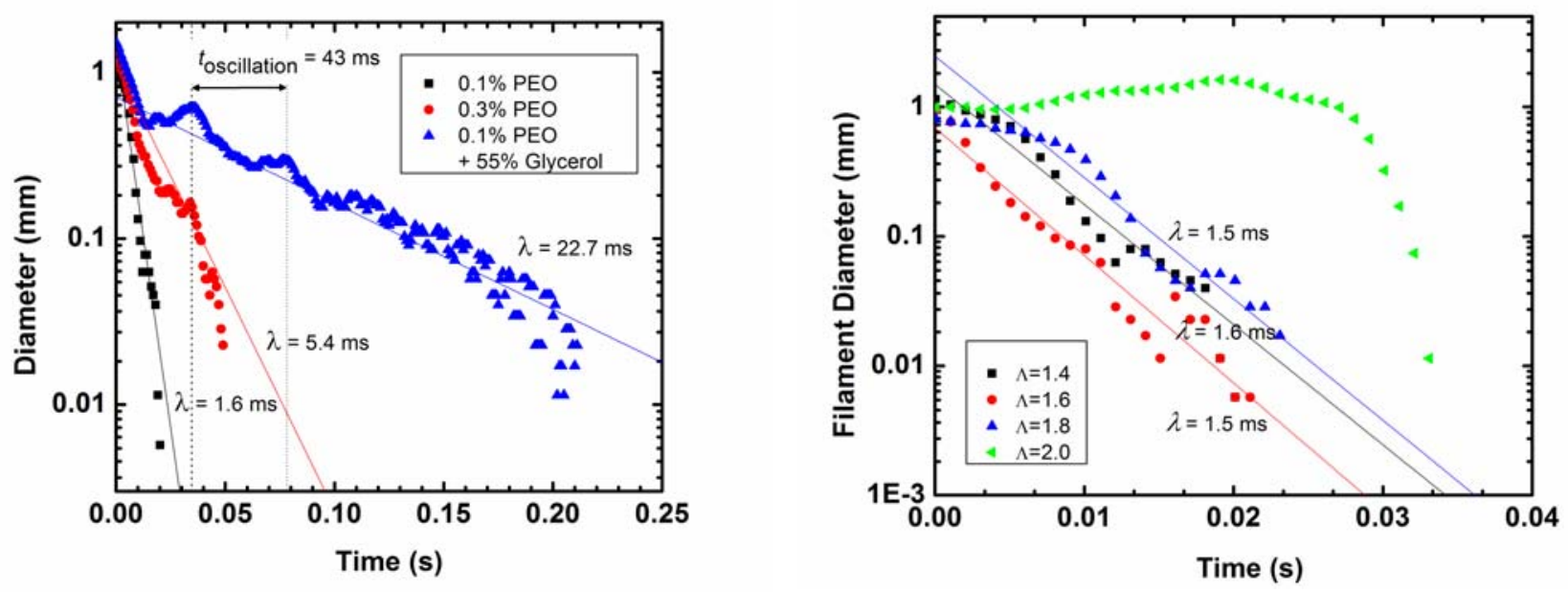

Figure 4. Exponential decay of fluid filament diameter for (a) $0.1 \%$ PEO, $0.3 \%$ PEO and $0.1 \%$ $\mathrm{PEO} /$ glycerol solutions at an aspect ratio of 1.41 , and (b) $0.1 \% \mathrm{PEO}$ solution for $h_{0}=3 \mathrm{~mm}$ and $\Lambda=1.41,1.61,1.79$ and 2.0. 


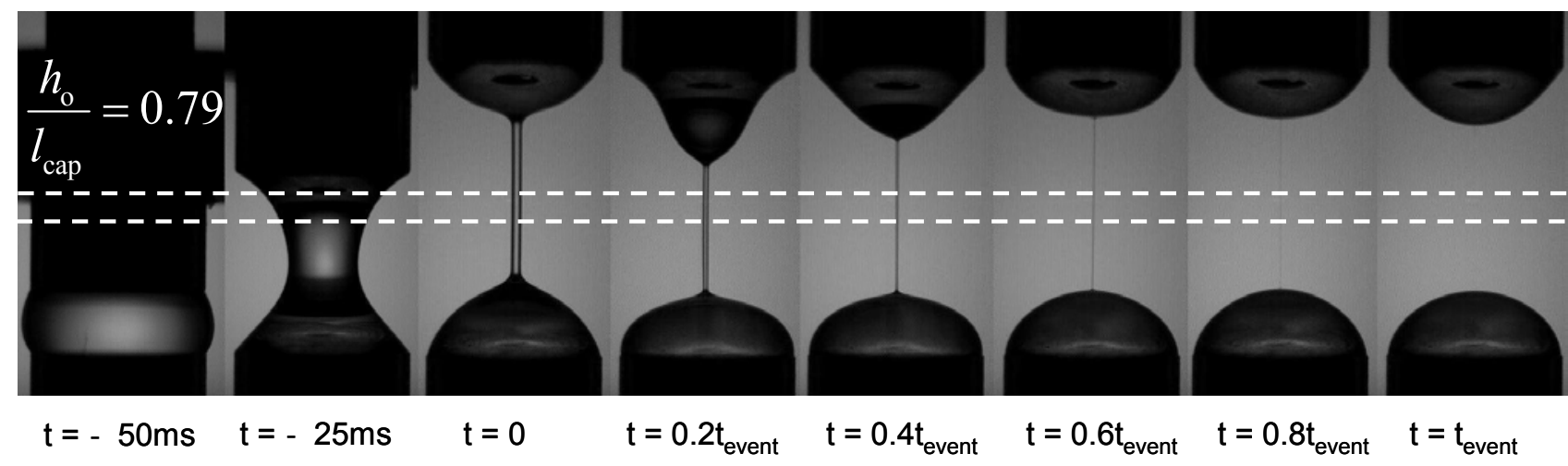

Figure 5. Filament thinning of the $0.3 \% \mathrm{PEO}$ solution for an initial gap height of $h_{\mathrm{o}}=2 \mathrm{~mm}$ and $\Lambda=1.61$, in which the total time of the event, $t_{\text {event }}=100 \mathrm{~ms}$

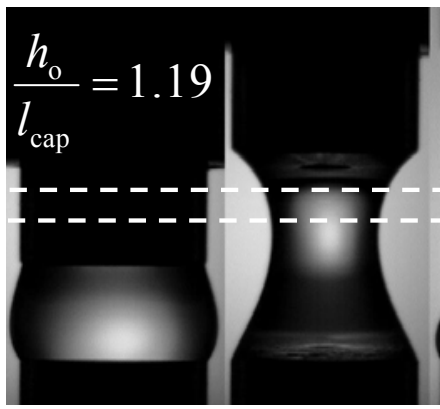

$\mathrm{t}=-50 \mathrm{~ms} \quad \mathrm{t}=-25 \mathrm{~ms}$

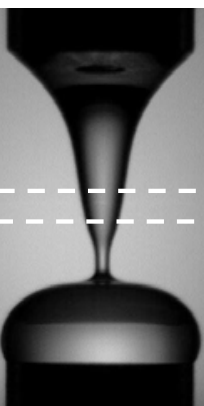

$t=0$

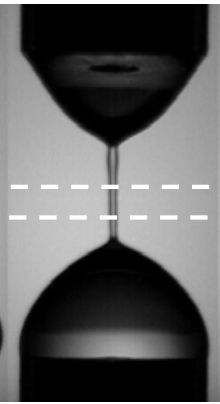

$\mathrm{t}=0.2 \mathrm{t}_{\text {event }}$

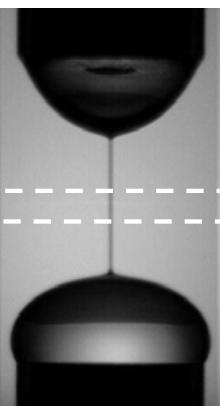

$\mathrm{t}=0.4 \mathrm{t}_{\text {event }}$

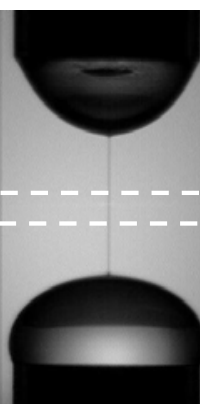

$\mathrm{t}=0.6 \mathrm{t}_{\text {event }}$

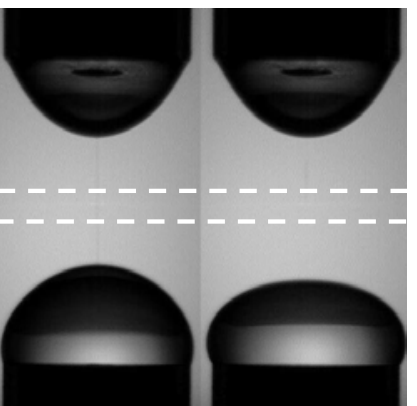

$\mathrm{t}=0.8 \mathrm{t}_{\text {event }} \quad \mathrm{t}=\mathrm{t}_{\text {event }}$

Figure 6. Filament thinning of the $0.3 \%$ PEO solution for an initial gap height of $h_{\mathrm{o}}=3 \mathrm{~mm}$ and $\Lambda=1.61$, in which the total time of the event, $t_{\text {event }}=110 \mathrm{~ms}$.

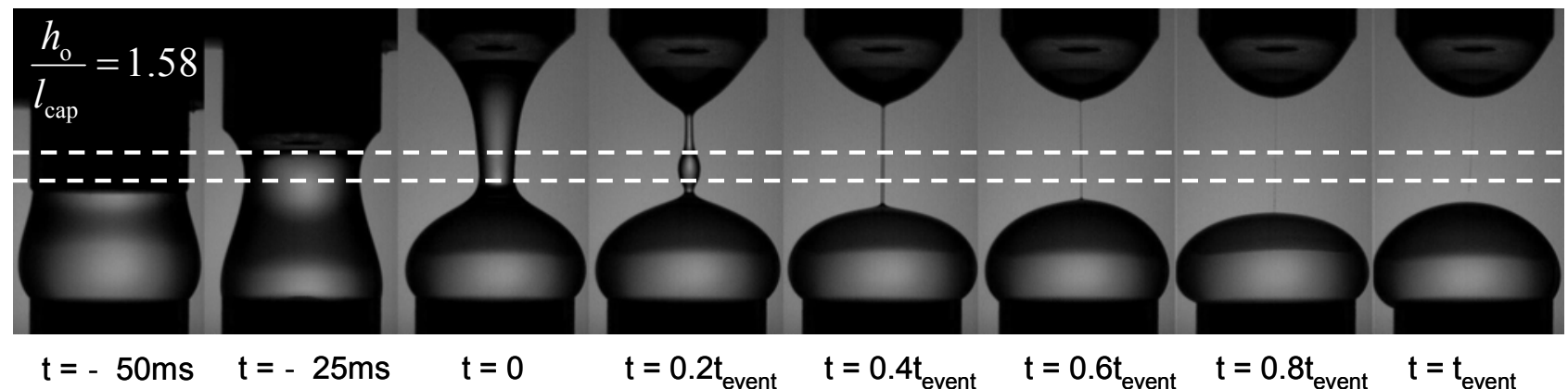

Figure 7. Filament thinning of the $0.3 \%$ PEO solution for an initial gap height of $h_{\mathrm{o}}=4 \mathrm{~mm}$ and $\Lambda=1.61$, in which the total time of the event, $t_{\text {event }} 125 \mathrm{~ms}$. 


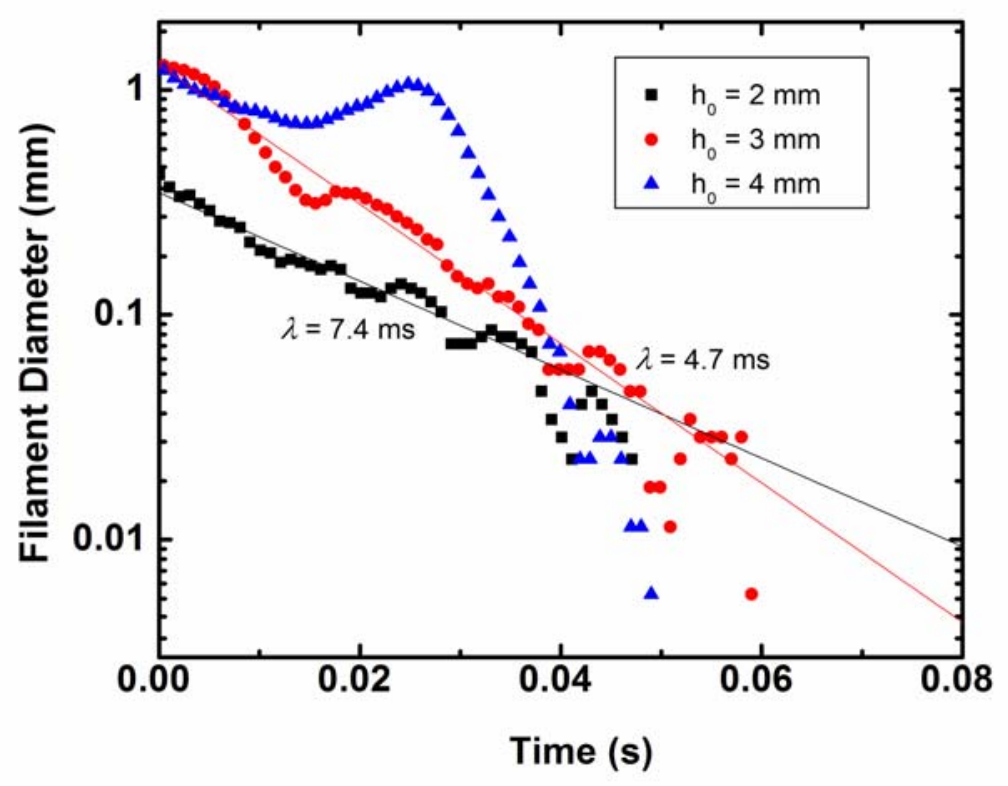

Figure 8. Exponential decay of the fluid filament diameter for the $0.3 \%$ PEO solution for $\Lambda=1.6$ and initial sample heights of $h_{0}=2,3$ and $4 \mathrm{~mm}$.

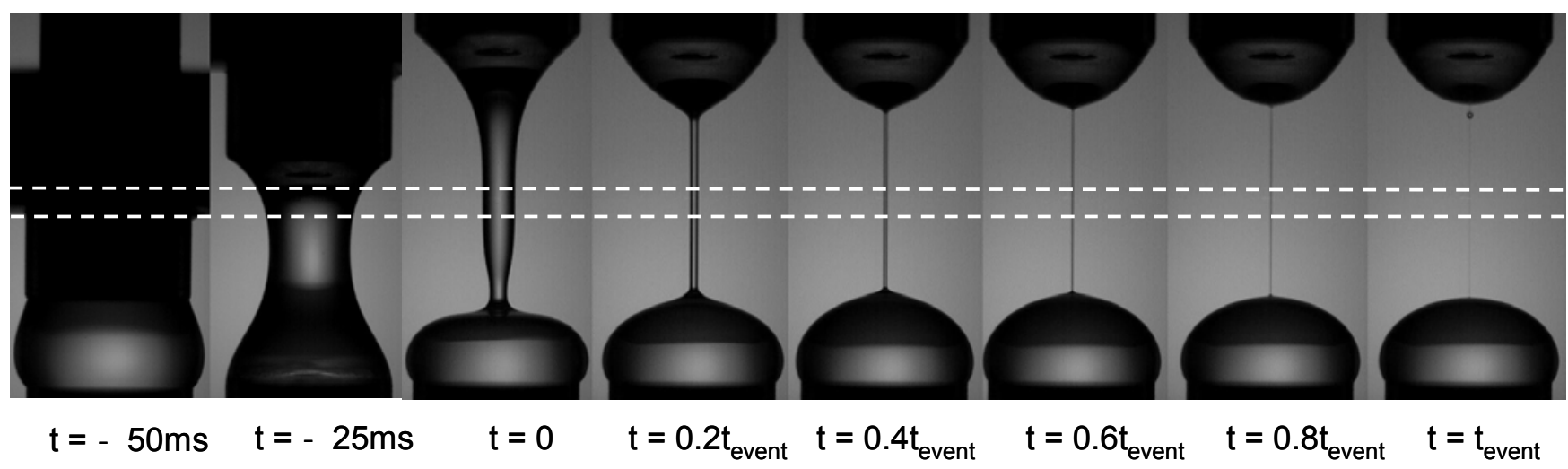

Figure 9. Thinning of fluid filament for the $0.1 \% \mathrm{PEO} /$ glycerol solution for $\Lambda=2.0$ and $h_{0}=3 \mathrm{~mm}$, in which the total event time, $t_{\text {event }}=420 \mathrm{~ms}$. 

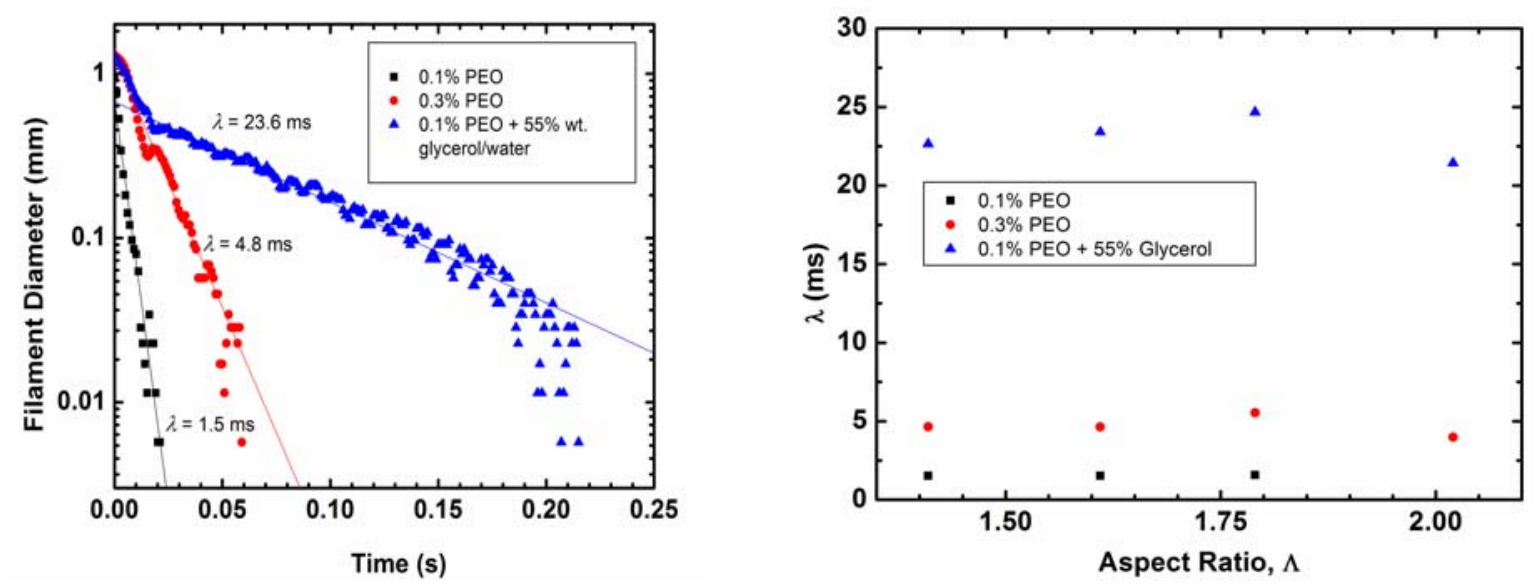

Figure 10. (a) Exponential decay of the fluid filament diameter for $\Lambda=1.61$ and $h_{0}=3 \mathrm{~mm}$ and (b) relaxation time as a function of aspect ratio, for the $0.1 \% \mathrm{PEO}, 0.3 \% \mathrm{PEO}$ and $0.1 \% \mathrm{PEO} / \mathrm{glycerol}$ solutions in which $h_{0}=3 \mathrm{~mm}$.

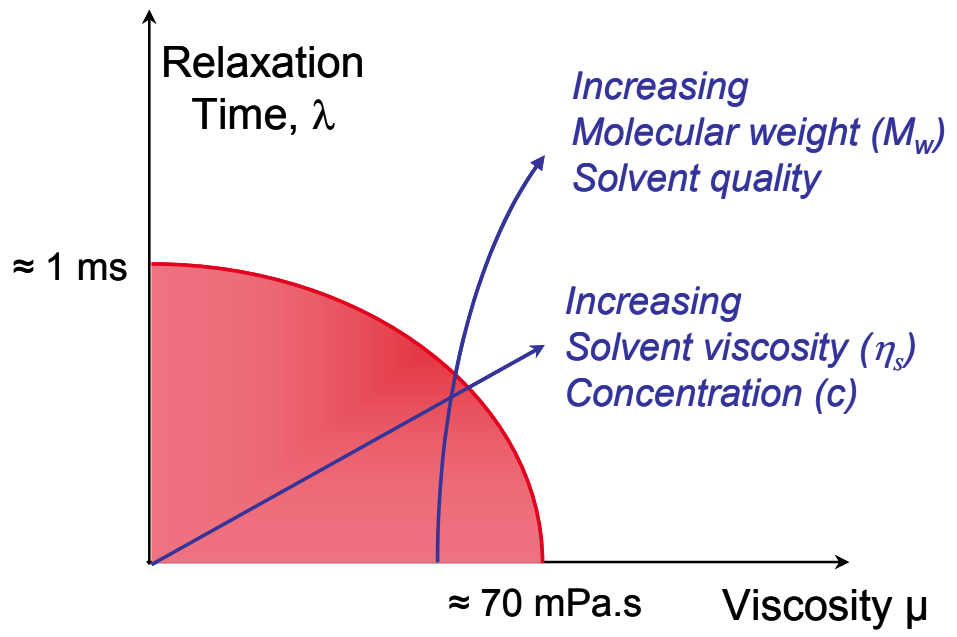

Figure 11. An operability diagram for capillary break-up elongational rheometry showing the minimum values of viscosity $(\mu)$ and relaxation time $(\lambda)$ required for successful measurement of the capillary thinning process. 